\title{
Estudio sobre el centro histórico de la Habana Vieja.
}

\author{
Study about Old Havana's historic center
}

Arlette Arceo Martínez. ${ }^{1}$, Adriel Malvarez Cuello. ${ }^{2}$ \& Luis Efraín Velasteguí López. ${ }^{3}$

\begin{abstract}
:
The tourist development, and his repercussion in different levels of the environment, they constitute an economic activity that deserves to be observed and measure with extreme precision for a projection guessed right toward future scenarios. The tourist destinations, containers of great quantity of resources and attractiveness, are one of the most complex forms, by their definition as maximum level of the tourist territorial product. Old Havana's historical center, in this condition, shows certain peculiarities that transform it into a consolidated destination and with marked tendency to the growth of their tourist demand. However, and in spite of their explotation level, it presents characteristic that could favor even more the favorable development of the destination.
\end{abstract}

Key words: Tourist Destination, growth, development, resources, attractiveness.

\section{Resumen:}

El desarrollo turístico, y la repercusión en los diferentes niveles del entorno, constituyen una actividad económica que merece ser observada y medida con extrema precisión para una proyección acertada hacia escenarios futuros. Los destinos turísticos, contenedores de gran cantidad de recursos y atractivos, son una de las formas más complejas dada su definición como máximo nivel del producto turístico territorial. El centro histórico de La Habana Vieja, bajo esta condición ostenta ciertas peculiaridades que lo convierten en un destino consolidado y con marcada tendencia al crecimiento de su demanda turística. Sin embargo, y a pesar de su nivel de explotación, presenta características que podrían favorecer aún más el desarrollo favorable del destino.

\footnotetext{
${ }^{1}$ Facultad de Turismo-Universidad de La Habana, Cuba, arceomartinezarlette@gmail.com

${ }^{2}$ Facultad de Turismo-Universidad de La Habana, Cuba, adrielmalva2017@gmail.com

${ }^{3}$ Ciencia Digital Editorial, Ecuador, luisefrainvelastegui@cienciadigital.org
} 
Palabras claves: Destino turístico, crecimiento, desarrollo, recursos, atractivos.

\section{Introducción.}

La Habana Vieja es el centro colonial más importante del Caribe y también uno de los más importantes de América. Se distingue por conservar un valioso patrimonio arquitectónico, además de tener unos pobladores caracterizados por su afabilidad, alegría y franca hospitalidad. A diferencia de las típicas ciudades coloniales, La Habana se desarrolló no sobre una, sino sobre cuatro plazas principales: La Plaza de Armas, la Plaza de la Catedral, la Plaza Vieja y la Plaza de San Francisco de Asís. El Centro Histórico de la Habana Vieja fue declarado Patrimonio Cultural de la Humanidad por la Organización de Naciones Unidas para la Educación, la Ciencia y la Cultura (UNESCO), en 1982 y posee atractivos únicos que lo han convertido en un sitio de preferencia, tanto para los cubanos como para los visitantes foráneos. Este, junto al sistema de fortificaciones se encuentra bajo protección legal y jurídica de las Leyes No. 1 y 2 de la Asamblea Nacional del Poder Popular, la Ley de la Protección al Patrimonio Cultural del 4 de agosto de 1977; Ley de los Monumentos Nacionales y Locales del 4 de agosto de 1977; y la Resolución No. 3 de la Comisión Nacional de Monumentos que declara al Centro Histórico de la antigua villa de San Cristóbal de La Habana como Monumento Nacional el 10 de octubre de 1978. El Centro Histórico ocupa el extremo noreste del municipio La Habana Vieja, un área de 2,14 km2 que abarca la zona de la antigua ciudad intramuros. El territorio limita al norte con la explanada de La Punta, por el este y el sur con el litoral de la bahía, y por el oeste con las calles Factoría, Cárdenas, Amistad, Industria y con el Paseo del Prado, quedando circunscritos cinco Consejos Populares: Catedral, Plaza Vieja, Belén, San Isidro, Prado y una porción del Consejo Jesús María. (Ecured.cu, recuperado 2017)

Este territorio puede clasificarse, por su ubicación geográfica como un espacio urbano y por su organización como una zona turística debido a que es un territorio con clara delimitación geográfica (por accidentes naturales: la bahía de La Habana y por las formas de acceso) en donde coinciden instalaciones vinculadas a atractivos de sitios, donde los clientes se desplazan en medios limitados de locomoción (individuales o por pequeños grupos) y estas están subordinadas a diferentes entidades.

Es indiscutible la importancia que posee este territorio para la conformación de la oferta turística a nivel de destino país, razón por la cual se hace necesario realizar un análisis minucioso sobre el desarrollo del turismo en el mismo. El presente trabajo es el resultado de una investigación efectuada en el Centro Histórico que tiene como objetivo general: Analizar el comportamiento de la actividad turística en el Centro Histórico, así como las potencialidades turísticas que posee para la confección de medidas o acciones para impulsar el desarrollo del turismo en el territorio.

\section{Metodología.}

Para la realización de dicha investigación se emplearon los siguientes métodos teóricos: 
1. Analítico sintético: Proporcionó desarrollar el análisis y la síntesis de la actividad turística en el centro histórico de La Habana, mediante el cual se descompone la misma en los principales elementos que la integran para analizar, valorar y conocer sus particularidades y simultáneamente a través de la síntesis integrarlos para verlos como un todo.

2. Lógico abstracto: Posibilitó aislar, separar y determinar las cualidades esenciales que caracterizaron al desarrollo del turismo en el destino.

3. Histórico - lógico: La utilización de este método permitió establecer la necesaria correspondencia entre los elementos de los métodos históricos y lógicos, con el fin de analizar la evolución histórica de la actividad turística en La Habana Vieja, con la proyección lógica de su desarrollo futuro.

Se utilizaron herramientas y métodos, reconocidos en el campo de la gestión de destinos turísticos tales como el Test de O’Meara y la medición de las 5 Fuerzas de Porter, vistas desde una perspectiva general del destino.

\section{Resultados.}

Descripción de las principales características del Centro Histórico de La Habana Vieja.

El recurso turístico puede ser definido como cualquier facilidad que sea, o pueda ser utilizada por los turistas, pero los turistas necesitan de una amplia variedad de facilidades aunque realmente usan muy pocas. Están constituidos por elementos naturales, históricos y culturales. (Fernández, 2014) Dentro de ellos, se destacan aquellos elementos que son considerados como patrimoniales, donde los objetos y lugares de carácter patrimonial son atractivos cada vez más valorados por la demanda turística actual. Por esto los órganos de gestión turística de un destino se preocupan por incorporar los componentes del patrimonio a su oferta. Una división simple de los recursos es la distinción entre primarios y secundarios. Los primarios son los que atraen al turista y son el principal motivo de su visita, y los secundarios constituyen otros recursos visitados durante la estancia. Esta distinción no implica cuantitativamente mayor intensidad de uso o de formas. Por el contrario, los visitantes vienen a las ciudades por sus atracciones primarias, pero gastan su tiempo y dinero en las facilidades secundarias, las cuales están más localizadas y se usan repetidamente; además, los primarios son creados o existen para el turismo, pero los secundarios no.

El centro Histórico de la Habana Vieja es un espacio de atracciones turísticas, pues conserva una extraordinaria riqueza de valores histórico-arquitectónicos y culturales, colorido y movimiento, una población local con sus hábitos y costumbres que le otorgan una identidad propia, convirtiéndolo en un destino de obligatoria visita que comprende recursos histórico-culturales, infraestructurales y socioeconómicos. Pero son los recursos histórico-culturales y patrimoniales los que más influyen en el desarrollo de la actividad turística en el territorio. (García)

El Centro Histórico de la Habana es rico culturalmente, posee atractivos únicos que lo han convertido en un sitio de preferencia, tanto para los cubanos como para los visitantes 
foráneos. (Mayoral) Cuenta actualmente según (Oficina Nacional de Estadídtica e Información de Cuba. , 2015) con:

- 24 museos y 9 casas museos

- 9 sitios históricos

- 9 iglesias

- 16 galerías de arte

- 3 teatros

- 3 librerías y bibliotecas

- 6 centros de promoción cultural

- 5 plazas principales

- 14 plazuelas y parques

Una mezcla de arquitectura y testimonio de diferentes épocas: monumentos antiguos, fortificaciones, iglesias, conventos, palacios, callejuelas, plazas; lo convierten en el conjunto colonial más rico de América Latina. Los lugares más atractivos y de interés se pueden dividir en los siguientes grandes grupos:

\section{Las fortificaciones o fortalezas antiguas:}

Existen 4 grandes fortificaciones en la Habana Vieja que se conservan con todos los detalles de la época en que los corsarios y piratas eran una amenaza en el Caribe: El Castillo de los Tres Reyes del Morro, que es el guardián de la bahía junto con el Castillo de San Salvador de La Punta, la Fortaleza San Carlos de la Cabaña con sus cañones apuntando a la Habana y el Castillo de la Real Fuerza con la ciudad a sus espaldas.

\section{El Paseo del Prado y áreas extramuros:}

La antigua ciudad estaba cercada por una muralla que le servía de protección. Aún se pueden ver algunos restos en la calle Egido. Siempre se ha considerado esta línea como la división que limita la Habana Vieja del resto de la ciudad, pero del otro lado hay edificaciones y monumentos que las unen por la historia, la cultura y la arquitectura. Todas se encuentran cerca del Paseo del Prado, una de las calles más famosas de la Habana, trazada de norte a sur, que llega desde el Malecón a la entrada de la bahía, hasta la Fuente de la India, en una esquina del Parque de La Fraternidad. En ella se encuentran ubicados el Capitolio de la Habana, el Parque Central, el Gran Teatro de La Habana, el mencionado Parque de la Fraternidad, La Fuente de la India, entre otros lugares de interés, incluyendo reconocidos hoteles de la zona.

\section{El área de la Plaza de la Catedral:}

La Plaza de la Catedral es un pequeño cuadrado frente a La Catedral de la Habana, la principal iglesia católica de la ciudad desde 1773, rodeada por antiguas edificaciones históricas. Algunas de ellas son: El restaurante El Patio (antigua casa del Marqués de Aguas Claras), El Museo de Arte Colonial (antigua casa de Don Luis Chacón o Conde de 
Casa Bayona), La antigua oficina del Historiador (antigua Casa del Conde Lombillo) y la Casa del Marqués de Arcos.

\section{El área de la Plaza de Armas:}

La Plaza de Armas fue el primer espacio público de la ciudad. Era el centro y el lugar más importante en la Habana colonial, por encontrarse a su alrededor las sedes de las autoridades civiles y militares en la época. La rodean actualmente las siguientes edificaciones: El Palacio de los Capitanes Generales (actual museo de la ciudad), El Palacio del Segundo Cabo (sede del Instituto Cubano del Libro), El Castillo de la Fuerza (actual museo naval), El Templete, El Hotel Santa Isabel (antigua casa de los Condes de Santovenia), el Museo de Historia Natural y la Biblioteca Rubén Martínez Villena (antigua embajada norteamericana). En el centro de la Plaza se encuentra la estatua de Carlos Manuel de Céspedes, en honor al Padre de la Patria.

\section{El área de la Plaza de San Francisco:}

La Plaza de San Francisco, famosa por sus palomas y la tercera fundada en la Habana, se encuentra a un lado del Convento y la Iglesia de San Francisco de Asís, templo fundado por esta orden religiosa en 1575, cuya torre fue por siglos el punto más alto de la Villa. A un lado se encuentra el edificio de la Lonja del Comercio con la famosa estatua de Mercurio (el Dios del Comercio) en su cúpula. Al frente, a la orilla de la bahía, la antigua Aduana y los muelles (actual Terminal de Cruceros Sierra Maestra). En el centro de la plaza se encuentra la Fuente de los Leones, una de las más bellas y de mayor simbolismo de la ciudad.

\section{El área de la Plaza Vieja:}

La Plaza Vieja es una de las más amplias de la ciudad, rodeada por muchas edificaciones históricas, sitio de residencias de duques, marqueses y personajes importantes de la época colonial. Actualmente tiene mucha vida cultural. Algunos de los edificios del lugar son: La Cámara Oscura (antiguo edificio de Gómez Vila), Las Casa de los Condes Jaruco (actual Casa de Cerveza), La Casa del Marqués de Casa Torres, y otros sitios de interés como: El Planetario.

\section{Las iglesias, parroquias y conventos.}

Los recursos turísticos culturales no se agotan con el patrimonio arquitectónico y urbanístico; la ciudad ofrece muchas posibilidades en relación con fiestas, eventos y programas culturales, entre otras, que son puestas en consideración para el mercado turístico.

El Centro Histórico de La Habana Vieja es una zona turística de tipo cultural cuyo principal atractivo lo constituye la monumentalidad y riqueza histórico-artística de su espacio, amparado por su condición de Patrimonio de la Humanidad otorgada en 1982 y por el compromiso de su población con el turismo. La labor del Centro Histórico de La Habana, dirigido por la Oficina del Historiador de la Ciudad de La Habana representa un 
ícono en el desempeño del turismo cultural sostenible. Durante casi tres lustros se han venido acumulando resultados socioeconómicos y culturales positivos, generando crecimiento y desarrollo en esta localidad. Lo cual es compatible con la conservación y resguardo del paisaje urbano, de múltiples tradiciones, de interculturalidad y de la ciudadanía que participa y se beneficia de los frutos del turismo. Ello sin dejar de realizar aportes para beneficio de todo el país. Una agencia de viajes propia, San Cristóbal de La Habana, es la encargada de operar el turismo histórico cultural de esta localidad y hoteles singulares alojan a los turistas, además de contar con la agencia Viajes Cubanacán, que dentro del turismo especializado atiende al turismo cultural, contando, entre otros, con recorridos por el Centro Histórico de la Habana Vieja.

Características del turismo cultural en el Centro Histórico según (Chávez, 2005)

- Es mayoritariamente un turismo de corta estancia en el destino, ya que comparte la visita con otros sitios y modalidades. La estancia media es reducida y existe gran cantidad de excursionistas que tan solo pasan unas horas en él.

- Es un turismo itinerante, de recorrido, lo que implica desplazamiento por el lugar; basa su acción en conocer sitios que al estar distribuidos en el territorio, generan rutas y recorridos para poder abarcarlos y conectarlos.

- La acción de los turistas está orientada al conocimiento y visita de lugares de interés cultural, artístico, histórico, y al consumo de servicios y productos con un fuerte carácter local.

- Al ser un turismo de paso, de corta estancia, la planta gastronómica y comercial adquiere más desarrollo que la planta de alojamiento, y los sitios de recreación tampoco alcanzan mayores dimensiones.

Por otra parte, la ciudad ofrece muchas posibilidades en relación con eventos y programas culturales, exposiciones y ferias, que de cierta forma también tributan a la modalidad de turismo de eventos, pero con un marcado carácter cultural. Algunas consideraciones que se han emitido con respecto a esta modalidad denotan que el turismo cultural continuará desarrollándose a la par con el crecimiento de la demanda, y que las ofertas se multiplicarán de igual modo. Otras expectativas se vinculan a que continúen creciendo, de ser posible, en más alta proporción los atractivos vinculados a la cultura popular y las tradiciones vivas e intangibles que son en general menos costosas de y una tendencia al perfeccionamiento de la promoción, la comercialización y la distribución internacional del turismo cultural, especialmente usando las nuevas tecnologías de la información y las comunicaciones y el vínculo directo turista prestador del servicio.

\section{Principales productos que conforman la oferta.}

Se define el producto turístico como: el conjunto de bienes y servicios necesarios para el desarrollo de la actividad turística. Estos se clasifican en Producto turístico "Viajes", Producto Turístico "Instalaciones" (atractivos y facilidades), Producto Turístico “Territorios". (Fernández, 2014) 
El producto turístico viajes, en este caso, se puede ver reflejado en numerosas excursiones y recorridos por el centro histórico, que ofertan varias agencias de viaje de gran prestigio. Por ejemplo, la agencia Viajes Cubanacán, San Cristóbal de la Habana, Cubatur y Havanatur, realizan varias excursiones, así como citytour tanto panorámicos como recorridos a pie por el centro histórico, que resaltan la belleza de su patrimonio cultural. Entre ellas: la excursión Mi Habana Colonial (Cubatur), excursión city tour Habana, Recorrido panorámico por La Habana Vieja, Recorridos por la Habana en autos antiguos, entre otros.

Como parte del producto turístico Instalaciones, se incluyen todos aquellos sitios, eventos o entidades que proveen de atractivos para motivar los viajes y también donde se brinden servicios y productos que faciliten la estancia1, aquí se pueden citar todos aquellos productos de hospedaje, gastronomía o restauración, comercio, y actividades complementarias. El Centro Histórico cuenta con una exquisita red hotelera, con opciones para los gustos más exigentes, que van desde pequeñas instalaciones enclavadas en mansiones coloniales, hasta hoteles que antaño fueron renombrados y renacieron en sus inmuebles originales, especializado en turismo de ciudad. Dispone de hoteles y hostales gerenciados por las cadenas hoteleras Gran Caribe, Cubanacán y Habaguanex. A continuación se disponen algunos de estos hoteles y hostales:

- Hostal los Frailes: hotel de Patrimonio, que reabrió en el año 2001, se encuentra en una calle adoquinada de La Habana Vieja (Teniente Rey), frente al convento de San Francisco de Asís. El bar del vestíbulo y el patio tranquilo con jardín hacen un lugar perfecto para relajarse lejos del bullicio.

- Hotel Ambos Mundos: es internacionalmente conocido porque es el hotel donde el Premio Nobel Ernest Hemingway se hospedó en Cuba y donde escribió la primera parte de su novela "Por quién doblan las campanas".

- Hotel Santa Isabel: situado en la Plaza de Armas, a pocos metros del lugar donde se fundó la ciudad. En 1867 el magnífico palacio del Conde de Santovenia se convirtió en el hotel favorito de los viajeros ilustres.

- Hotel Saratoga: es una elegante construcción con tendencia ecléctica de estilo neoclásico, con espectaculares vistas del Capitolio, el Parque de la Fraternidad, la prestigiosa Fábrica de tabacos Partagás y el Gran Teatro de La Habana.

- Hotel Palacio de San Felipe y Santiago de Bejucal: es el primer hotel de Habaguanex en la Plaza San Francisco de Asís. Historia y modernidad se unen en este edificio que a finales del siglo XVIII era propiedad de don Sebastián de Peñalver.

- Hotel Iberostar Parque Central: es uno de los hoteles internacionales más lujosos de la ciudad, ofreciendo todas las facilidades necesarias. Su estilo arquitectónico moderno, en cierta medida, aún conserva ciertos rasgos de los edificios coloniales que se encuentran en La Habana Vieja.

- Hostal Conde de Villanueva: está dedicado a los amantes del tabaco, que pueden comprar las mejores marcas de puros habanos en su Casa del Habano, y disfrutar 
del placer de fumar en su salón privado. El edificio fue la residencia de Claudio Martínez de Pinillos, Conde de Villanueva.

Por otra parte, una amplia red extrahotelera, con restaurantes, boutiques y tiendas, entre otras, es complemento necesario para turistas y citadinos. La red gastronómica está especializada en comida árabe, italiana, criolla, internacional, española, oriental, de mariscos, mexicana, de cocina dietética y vegetariana, y heladerías. La red comercial incluye boutiques y centros comerciales. Algunos de los principales restaurantes que se encuentran en el territorio son:

- Restaurante Bodegón Onda

- Restaurante El Templete

- Restaurante Café Taberna

- Restaurante La Imprenta

- Restaurante Castillo de Farnés

- Restaurante La Mina

- Restaurante El Baturro

- Restaurante La Bodeguita del Medio

- Restaurante El Mesón de la Flota

- Restaurante Jardín del Edén

- Restaurante El Patio

- Restaurante El Mercurio

- Restaurante Factoría Plaza Vieja

- Restaurante Cantabria

- Restaurante Santo Ángel

- Restaurante Café del Oriente

Otros productos turísticos presentes en el territorio son todos aquellos que han surgido como parte de la apertura en el país del sector no estatal, naciendo así casas de renta y restaurantes (comúnmente llamados "paladares"), que contribuyen al aumento de la oferta turística en el Centro Histórico.

La actividad turística en el Centro Histórico de La Habana se manejaba fundamentalmente por dos empresas o entidades turísticas, la Compañía Turística Habaguanex (actualmente desaparecida) y la Agencia de Viajes San Cristóbal S.A.

La Agencia de Viajes San Cristóbal es reconocida como la única agencia receptiva cubana especializada en turismo histórico-cultural y patrimonial, que promueve estos valores del destino Cuba en los principales mercados emisores a través de una carpeta de productos turísticos altamente especializados, donde se combinan elementos del patrimonio tangible e intangible de la capital cubana con el resto de las ciudades históricas y patrimoniales del país, así como reservas naturales y otros sitios de interés turístico. Desde 1994 administra hoteles singulares asociados al Centro Histórico, así como otras actividades de alimentos y bebidas y comerciales en esta localidad. (Ecured. cu, 2017) 
La agencia cuenta con un equipo de guías de alto nivel profesional, (premisa indispensable para el trabajo que se realiza en la Agencia) seleccionados intencionalmente a partir de su calificación (generalmente graduados de la Facultad de Lenguas Extranjeras de la Universidad de La Habana y con dominio de dos o tres idiomas cada uno). Para ellos se desarrolla una estrategia de habilitación y capacitación directa en la que intervienen directivos de primer nivel de las principales entidades de la Oficina del Historiador de la Ciudad de La Habana. Esta tarea contribuye a alcanzar el alto nivel de preparación en historia, cultura, patrimonio y educación ambiental, pero también a su sensibilización y compromiso con el proyecto del que forman parte.

La experiencia acumulada con otras casas de incentivos le permite a la Agencia de Viajes San Cristóbal ofrecer la logística y organización de paquetes de incentivos mediante programas de motivación, dinámicas de grupos y visitas turísticas con un producto a elegir. Se encarga también de brindar propuestas tanto para clientes individuales como para grupos, de programas de corta o larga estancia, especializados en temas arquitectónicos, históricos, culturales, religiosos, entre otros. Así como programas que incluyen temas relacionados con el habano y el ron. (Guilarte, 2015)

Otra vía de reactivación y diversificación económica del Centro Histórico es el sector inmobiliario que dirige como accionista principal Fenix SA, creada en 1996, para arrendar inmuebles como viviendas para estancias de larga duración, oficinas, locales comerciales, alquiler de coches comerciales, comercialización de piezas y accesorios para automóviles, todo ello tributando ingresos económicos para la gestión del casco histórico. Otra variante incorporada es el turismo de cruceros, restaurando el primero de los muelles para el atraque de cruceros en el entorno de la Plaza de San Francisco.

\section{Ciclo de Vida del Destino turístico.}

El ciclo de vida del destino turístico constituye uno de los temas de mayor relevancia en los análisis de competitividad turística. Muestra la evolución de un destino en el tiempo y puede ayudar a identificar la estrategia más coherente a llevar a cabo. (Baro, 2017).

Teniendo en cuenta dimensiones relacionadas con las infraestructuras, actitudes de los turistas y residentes, implicación de agentes locales y externos en la comercialización del producto, accesibilidad a los destinos y competencia de los mismos, en el presente trabajo se analizaron como principales indicadores para el análisis del ciclo de vida de los destinos el número de visitantes y la evolución de la demanda, la competencia en el mercado y la comercialización de la oferta en el territorio. Tomando como punto de partida el primer indicador, la evolución de la demanda, y analizando el comportamiento de la cantidad de turistas-días en el territorio, en el período comprendido entre los años 2014 y 2018 se puede observar que la demanda mantuvo un crecimiento sostenido, que, si bien se desarrolló aceleradamente en los primeros años del período analizado, disminuyó su velocidad, mostrando un aumento relativamente discreto en los años postreros. (Castro, 2014) 
Para el análisis de la competencia se emplearon algunas de las fuerzas competitivas elaboradas por el profesor Michael Porter de la Harvard Business School en 1979. La rivalidad entre los competidores se podría catalogar de alta, si se toman como referencia otros espacios turísticos del destino La Habana que gozan también de gran popularidad entre los visitantes (Vedado, Miramar y Playas del Este) y destinos turísticos consolidados o en vías de desarrollo a lo largo del país como: Varadero, Trinidad, Cayería Norte de Villa Clara, Ciego de Ávila y Camagüey, Holguín y Santiago de Cuba (prestando especial atención a aquellos que promueven el turismo cultural o patrimonial). Por otro lado la amenaza de entrada de nuevos competidores y de productos o servicios sustitutos es media por todos aquellos destinos en crecimiento, en el país, en los que se pretende realizar proyectos de inversión, o que ya están en ejecución. Para analizar la comercialización y promoción de los productos turísticos que ofrece el Centro Histórico de La Habana se estudió la labor realizada por la Compañía Habaguanex y la Agencia de Viajes San Cristóbal. La venta de los servicios turísticos de la Agencia San Cristóbal se realiza a través de su oficina en el Centro Histórico de La Habana; de otras agencias receptivas nacionales como Havanatur, Cubanacán y Cubatur; mediante alianzas en el exterior; y utilizando alrededor de 200 tour operadores, fundamentalmente europeos. La agencia ha creado alianzas en diferentes países que hacen uso de la marca para la comercialización del producto, ellas son: San Cristóbal París-Pratt, San Cristóbal Italia, San Cristóbal Alemania-Suiza y Austria/Erlebe-Reisen GmbH, San Cristóbal UK- Old Havana Ltd., San Cristóbal Asia-Cuseko Travel, San Cristóbal España-PortugalClickHabana, y San Cristóbal Chile-Caribbean Chile S.A.

La página web de la agencia está a la disposición de los clientes con las ofertas de excursiones, programas y otros servicios de la agencia, desde la cual es posible la reserva de las excursiones, aunque no del resto de programas. Del análisis en conjunto de las páginas webs de San Cristóbal, hay que destacar su poca funcionalidad, con lo cual no se adaptan a las necesidades que tienen los usuarios de Internet. En ninguno de los casos es posible realizar la compra de los servicios y sólo en algunos es posible la reserva. El hecho de que no estén disponibles los precios y que sea necesario solicitar por email la información o llamar por teléfono, es un inconveniente importante en una sociedad en la cual las personas están cada vez más acostumbradas a las compras por Internet, por la rapidez y la comodidad que esto significa. En cuanto a las acciones de promoción hay que destacar que en Cuba las campañas publicitarias turísticas se realizan a nivel nacional. De este modo, desde 2010 el destino utiliza el slogan Auténtica Cuba. El vídeo promocional de la campaña (www.autenticacuba.com) dura algo más de 6 minutos y muestra no sólo los recursos naturales, sino la cultura y modo de vida de los cubanos. Por otra parte la Agencia San Cristóbal y la compañía Habaguanex cuentan con folletos promocionales sobre sus hoteles y la oferta de excursiones, programas y eventos culturales que comercializan. Sin embargo, lo importante es en qué medida estos folletos llegan hasta los visitantes. La oferta cultural de la Habana Vieja también se promociona mediante la Revista Opus Habana (www.opushabana.cu) y la emisora Habana Radio (www.habanaradio.cu). Ambas pertenecen a la $\mathrm{OHCH}$, y además de difundir la 
programación cultural también hacen eco de la labor de conservación y restauración del patrimonio que se lleva a cabo. En cuanto a la participación en ferias, hay que mencionar que San Cristóbal asiste, como parte del stand de Cuba, a las principales que tienen lugar en el sector turístico internacional. Por ejemplo, asiste a las ediciones de FITUR; ITB; World Travel Market; y la Feria de Turismo, Arte y Cultura de América Latina y Europa (EUROAL).

Luego del análisis realizado anteriormente se pasó a concluir que el Centro Histórico de La Habana Vieja se encuentra en la etapa de Consolidación, la cual se caracteriza por el crecimiento del número de turistas, pero no de forma tan exponencial como en la fase anterior. El destino ya forma parte de la industria organizada. Por la saturación de la demanda del producto tradicionalmente ofertado, los turistas iniciales se han desplazado a otros destinos. Aparecen destinos sustitutivos, y una masificación del espacio. Los destinos que se encuentran en esta fase han de realizar acciones de comercialización para conseguir el mantenimiento de su demanda y la revitalización de la oferta.

\section{Presencia de recursos turísticos no utilizados.}

Independientemente de la existencia de proyectos inversionistas que implican una renovación capital en esta zona con un consecuente desarrollo y aprovechamiento de los recursos turísticos, aún persisten en el territorio algunos recursos que son subutilizados y otros con acceso limitado, así como algunos que no son reconocidos. La Alameda de Paula constituye uno de los recursos subutilizados, así como el parque del mismo nombre. Entre los que no son reconocidos se encuentran algunos importantes sitios históricos como los restos de la Muralla y en el caso de los de difícil acceso están Jardines Diana de Gales y Madre Teresa y la Plazuela de Paula. Es necesario destacar la necesidad del aprovechamiento de dichos recursos pues su correcta explotación implicaría mayores beneficios para el sector turístico en la zona a partir de la diversificación de la oferta del centro histórico.

\section{Principales dificultades que entorpecen el desarrollo del turismo.}

De manera general y teniendo en cuenta algunas de las opiniones y señalamientos realizados por la población residente en el Centro Histórico de La Habana Vieja, en las encuestas, realizadas por estudiantes de la Facultad de Turismo de la Universidad de La Habana, como parte de la evaluación del impacto del turismo en la población proyectada por el Plan maestro de la Oficina del Historiador, a continuación se disponen algunas problemáticas o dificultades que obstaculizan o afectan el desarrollo del turismo en el territorio:

- Varias de las calles y edificios de la ciudad presentan un alto grado de deterioro.

- Existe insuficiente señalización vial.

- Existencia de suciedad en calles y plazas.

- Escasa iluminación en los viales, en algunos monumentos y edificios significativos. 
- Insuficiencias en los carros de renta.

- Insuficiencias en la variedad de productos en las tiendas.

- Los problemas para acceder a Internet en el destino debido a su elevado precio para los turistas o a la lentitud con que se navega en la red. Esta situación evidentemente representa un importante inconveniente para los visitantes, pero además limita la difusión de la oferta cultural que se realiza desde la web de la Oficina del Historiador de la Ciudad de la Habana $(\mathrm{OHCH})$.

- Las entidades gestoras del turismo en la Habana Vieja no emplean suficientemente las tecnologías de la información y la comunicación en la comercialización y promoción de la oferta cultural.

- Estimular la recopilación de materia prima y el reciclaje de los desechos sólidos en general, mediante la creación de centros que se dediquen a esta actividad en sus diversas modalidades.

- Escaso interés cultural que posee la población local sobre la historia del centro histórico.

Epígrafe 4: Medidas o acciones que deben implementarse para impulsar el desarrollo del turismo.

Partiendo de las dificultades que entorpecen el desarrollo del turismo y de lo investigado en este trabajo, deben tenerse en cuenta varias acciones que de implementarse impulsarían el desarrollo del turismo en el Centro Histórico de la Habana Vieja, entre ellas se pueden citar:

- Hacer un mejor uso de las TIC para llegar a un mayor número de clientes, mejorar la experiencia turística, y sobre todo para realzar su patrimonio cultural, tanto material como inmaterial.

- Implementar programas dirigidos a elevar la calidad de las áreas residenciales sobre la base de un planeamiento gestionable y un modelo de intervención acorde con las particularidades y requerimientos del territorio.

- Incentivar a la población residente a contribuir con la higienización de las calles y parques.

- Rescatar la red comercial y de servicios generales del territorio, en cuanto al servicio que prestan, aumentando y diversificando su oferta gastronómica y comercial.

- Estimular la participación de la población en actividades culturales, a partir del trabajo mancomunado de las diversas instalaciones culturales que funcionan en el territorio.

- Recuperar el estado técnico de los pavimentos y la señalización vertical y horizontal establecida.

- Implementar un programa de iluminación, con finalidad pública y/o de realce, de todos los espacios públicos del centro histórico, como son: las vías de mayor flujo 
vehicular, los corredores entre plazas, las calles de interés especial, las calles secundarias, las plazas, las plazuelas, y los parques.

- Estimular la inversión en los sistemas de acueducto, drenaje y alcantarillado, con el fin de garantizar la cantidad y calidad en el suministro de agua, así como un tratamiento adecuado de los residuos líquidos.

- Dotar de equipamiento adecuado que facilite la recogida de desechos sólidos.

\section{Conclusiones}

Luego de realizada la investigación pertinente sobre los temas que conformaron el presente trabajo, así como el análisis y comprensión de los resultados arrojados y la información obtenida con dicha investigación, se pudo arribar a las siguientes conclusiones:

- El Centro Histórico de La Habana Vieja cuenta con potencialidades y recursos turísticos varios, en especial históricos-culturales y patrimoniales para el desarrollo del turismo cultural; aunque esto no elimina la posibilidad de que se implementen otras modalidades, como el turismo de cruceros, que en la actualidad se ha incrementado su desarrollo significativamente.

- Cuenta con variada cantidad de recursos turísticos especialmente históricos culturales que hacen única su oferta y cuenta con una red hotelera y gastronómica que la respalda.

- El análisis del comportamiento de la actividad turística en el territorio, logró identificar como etapa del ciclo de vida de los destinos en que se encuentra, a la consolidación dada el comportamiento actual de la demanda, el crecimiento del número de competidores y algunas brechas en la comercialización de las ofertas turísticas del espacio turístico.

- A pesar de que su desarrollo turístico se encuentra madurez, existen algunas problemáticas que entorpecen el pleno desarrollo del turismo en la zona, y que de corregirse impulsarían este desarrollo, evitando un futuro estancamiento y declive del destino.

- El desempeño del Centro Histórico, aprovechando los beneficios del turismo cultural que se desarrolla, es una muestra indiscutible de turismo sostenible, que ha venido acumulando durante más de tres lustros efectos económicos y socioculturales positivos y contribuyendo a generar crecimiento y desarrollo en esta localidad.

\section{Referencias Bibliográficas}

Baro, M. (13 de enero de 2017). Hosteltur.com. Obtenido de Ciclo de Vida de los Destinos Turísticos.: http://comunidad.hosteltur.com/index.html 
Castro, H. A. (2014). Evolución de Indicadores Turísticos de Cuba 2011-2013. . En En Retos Turísticos Vol.2. La Habana.

Chávez, E. S. (2005). Turismo y desarrollo sostenible: el caso del centro histórico de La Habana, Cuba. Pasos: Revista de Turismo y Patrimonio Cultural.

Ecured. cu. (enero de 2017). Obtenido de Agencia San Cristóbal. : https://www.ecured.cu Ecured.cu. (recuperado 2017). Obtenido de La Habana Vieja. : https://www.ecured.cu Fernández, R. M. (2014). Principios, organización y práctica del turismo. La Habana: Félix Varela.

García, A. (s.f.). radiociudadhabana.icrt.cu . Obtenido de La bahía de La Habana: turismo y desarrollo local: http://www.radiociudadhabana.icrt.cu

Guilarte. (2015). La gestión del turismo cultural en La Habana Vieja. International Journal of Scientific Management and Tourism.

https://www.ecured.cu. (s.f.). Obtenido de Compañía Turística Habaguanex S.A. : https://www.ecured.cu

Mayoral, M. J. (s.f.). www.nacionyemigracion.cu. Obtenido de Inversiones turísticas en La Habana, florece una ciudad maravilla. : http://www.nacionyemigracion.cu/

Oficina Nacional de Estadídtica e Información de Cuba. . (2015). Obtenido de Anuario Estadístico La Habana Vieja.

Suárez, M. Á. (Recuperado el 18 de Enero de 2017). Etapas de la vida en un destino turístico. Obtenido de http://www.turiscom.org/

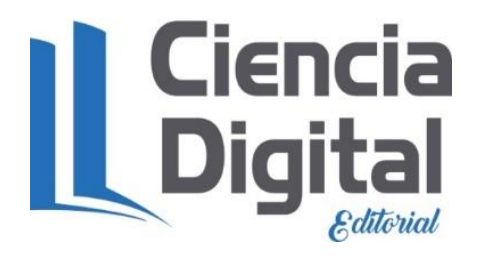




\section{PARA CITAR EL ARTÍCULO INDEXADO.}

Arceo Martínez, A., Malvarez Cuello, A., \& Velasteguí López, L. E. (2021). Estudio sobre el centro histórico de la Habana Vieja. Explorador Digital, 5(2), 120-134. https://doi.org/10.33262/exploradordigital.v5i2.1690

\section{¿Ciencia}

El artículo que se publica es de exclusiva responsabilidad de los autores y no necesariamente reflejan el pensamiento de la Revista Explorador Digital.

El artículo queda en propiedad de la revista y, por tanto, su publicación parcial y/o total en otro medio tiene que ser autorizado por el director de la Revista Explorador Digital.
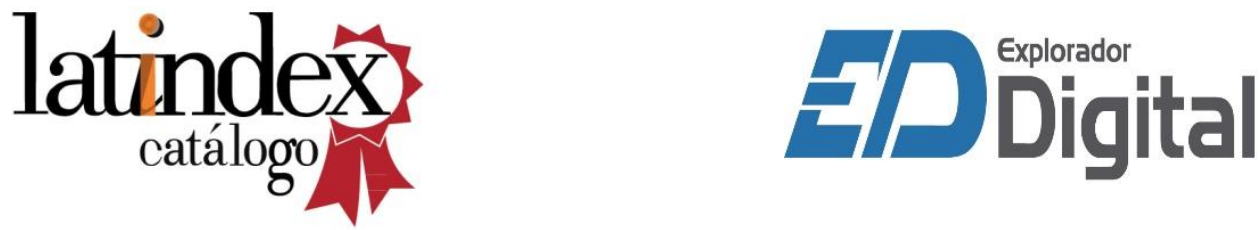